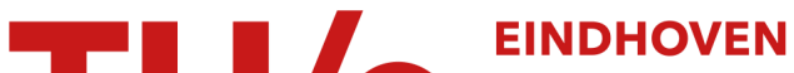 UNIVERSITY OF TECHNOLOGY
}

\section{Workload distributions in ASIP queueing networks}

\section{Citation for published version (APA):}

Boxma, O., Kella, O., \& Yechiali, U. (2021). Workload distributions in ASIP queueing networks. Queueing Systems, 97(1-2), 81-100. https://doi.org/10.1007/s11134-020-09678-4

\section{Document license:}

TAVERNE

DOI:

10.1007/s11134-020-09678-4

Document status and date:

Published: 01/02/2021

\section{Document Version:}

Publisher's PDF, also known as Version of Record (includes final page, issue and volume numbers)

\section{Please check the document version of this publication:}

- A submitted manuscript is the version of the article upon submission and before peer-review. There can be important differences between the submitted version and the official published version of record. People interested in the research are advised to contact the author for the final version of the publication, or visit the $\mathrm{DOI}$ to the publisher's website.

- The final author version and the galley proof are versions of the publication after peer review.

- The final published version features the final layout of the paper including the volume, issue and page numbers.

Link to publication

\section{General rights}

Copyright and moral rights for the publications made accessible in the public portal are retained by the authors and/or other copyright owners and it is a condition of accessing publications that users recognise and abide by the legal requirements associated with these rights.

- Users may download and print one copy of any publication from the public portal for the purpose of private study or research.

- You may not further distribute the material or use it for any profit-making activity or commercial gain

- You may freely distribute the URL identifying the publication in the public portal.

If the publication is distributed under the terms of Article 25fa of the Dutch Copyright Act, indicated by the "Taverne" license above, please follow below link for the End User Agreement:

www.tue.nl/taverne

Take down policy

If you believe that this document breaches copyright please contact us at:

openaccess@tue.nl

providing details and we will investigate your claim. 


\title{
Workload distributions in ASIP queueing networks
}

\author{
Onno Boxma ${ }^{1} \cdot$ Offer Kella $^{2}\left(\mathbb{D} \cdot\right.$ Uri Yechiali $^{3}$
}

Received: 3 June 2020 / Revised: 6 November 2020 / Accepted: 17 November 2020 /

Published online: 2 January 2021

(c) The Author(s), under exclusive licence to Springer Science+Business Media, LLC part of Springer Nature 2021

\begin{abstract}
The workload of a generalized $n$-site asymmetric simple inclusion process (ASIP) is investigated. Three models are analyzed. The first model is a serial network for which the steady-state Laplace-Stieltjes transform (LST) of the total workload in the first $k$ sites $(k \leq n)$ just after gate openings and at arbitrary epochs is derived. In a special case, the former (just after gate openings) turns out to be an LST of the sum of $k$ independent random variables. The second model is a 2-site ASIP with leakage from the first queue. Gate openings occur at exponentially distributed intervals, and the external input processes to the stations are two independent subordinator Lévy processes. The steady-state joint workload distribution right after gate openings, right before gate openings and at arbitrary epochs is derived. The third model is a shot-noise counterpart of the second model where the workload at the first queue behaves like a shot-noise process. The steady-state total amount of work just before a gate opening turns out to be a sum of two independent random variables.
\end{abstract}

Keywords ASIP queueing networks · Lévy networks · ASIP with leakage · ASIP queues in series · Workload

Onno Boxma: Research partly funded by an NWO TOP Grant, Grant No. 613.001.352, and by the NWO Gravitation project NETWORKS, Grant No. 024.002.003. Offer Kella: supported in part by Grant 1647/17 from the Israel Science Foundation and the Vigevani Chair in Statistics.

$凶$ Offer Kella

offer.kella@huji.ac.il

Onno Boxma

o.j.boxma@tue.nl

Uri Yechiali

uriy@tauex.tau.ac.il

1 EURANDOM and Department of Mathematics and Computer Science, Eindhoven University of Technology, P.O. Box 513, 5600 MB Eindhoven, The Netherlands

2 Department of Statistics, The Hebrew University of Jerusalem, 91905 Mount Scopus, Jerusalem, Israel

3 Department of Statistics and Operations Research, School of Mathematical Sciences, Tel Aviv University, 69978 Tel Aviv, Israel 
Mathematics Subject Classification Primary: 60K20 . 60K25 . 60K30 . Secondary: 60G51

\section{Introduction}

A tandem stochastic network is a linear set of $n$ sites (queues) denoted $Q_{1}, Q_{2}, \ldots, Q_{n}$ to which a random stream of particles (or work) flows. Every site consists of a buffer and a gate behind it that opens according to some stochastic process. Each site is characterized by some buffer capacity $C_{\text {site }}$, denoting the maximal number of particles (amount of work) that the buffer can hold, and by $C_{\text {gate }}$, the maximal number of particles (work) that can pass through the gate when it opens. Particles (work) flow into the system, usually to the first site, and then move unidirectionally from one site to the next, until exiting the system. Three fundamental models, distinguished by their $C_{\text {site }}$ and $C_{\text {gate }}$ values, have been analyzed in the literature: The first is a tandem Jackson network (TJN), where particles flow into the first site according to a Poisson process. The site capacities are $C_{\text {site }}=\infty$ and $C_{\text {gate }}=1$, while each gate opens independently every exponentially distributed period of time, allowing at most a single particle (if any) to hop to the next site. The TJN $[11,12]$ is famous for its product-form solution for the steady-state joint distribution function of the queue occupancies. The second model is the asymmetric exclusion process (ASEP), a fundamental model in nonequilibrium statistical physics [10,15], where $C_{\text {site }}=1$ and $C_{\text {gate }} \geq 1$. If the gate of $Q_{i}$ opens while the buffer of $Q_{i+1}$ is not empty, the particle in $Q_{i}$ is blocked. The third setup is the recently introduced [16,17,19] asymmetric inclusion process (ASIP), where both $C_{\text {site }}=\infty$ and $C_{\text {gate }}=\infty$. As such, the ASIP fills the missing link between the TJN and the ASEP. The major difference between the models is that in the ASIP, when the gate of $Q_{i}$ opens, all particles (work) present there move simultaneously and instantaneously to the buffer of the next site, joining the cluster of particles (work) there to form a larger cluster, while in the TJN or ASEP at most one particle moves forward when the site's gate opens. The ASIP may be considered as an inclusion counterpart of the ASEP and as a batch-service counterpart of the TJN. It was shown in [17] that, in contrast to the TJN, the ASIP does not admit a product-form solution for its steady-state joint distribution function of the queue occupancies. However, it admits a product-form solution for the site loads. ASIP's limit laws were treated in [18] and [19]. It was shown in [19] that, in a symmetric ASIP, the asymptotic probability that site $k$ is occupied is proportional to $1 / \sqrt{k}$. Occupation probabilities were further studied in [20]. The ASIP has been generalized in [6] to the case of general gate opening intervals, where gate openings are determined by a Markov renewal process. The focus in [6] is on the steady-state joint distribution function of the number of particles in the various sites. A very recent study [4] analyzed occupancy correlations in the classical ASIP.

The current paper focuses on the analysis of workload in a few tandem ASIP networks. Our motivation for this study is the following: Firstly, we wish to advance the knowledge of ASIP networks, as an important class of networks in between classical queueing networks and ASEP networks. Secondly, the tandem model that we treat in Sect. 2 is motivated by its applicability to statistical physics, as outlined in [17- 
20]. Thirdly, we want to add the feature of leakage to ASIP networks: Work not only leaves a site instantaneously at gate openings, but it also leaks gradually to the next site and/or the outside, whenever its queue is not empty. This makes such ASIP models significantly more complicated (accordingly, in Sects. 3 and 4 we restrict ourselves to two-queue ASIP models with leakage) but also significantly more versatile. Leakage could be viewed as regular service, whereas the gate openings could represent incidental clearings of the work that is left. Such phenomena may occur, for instance, in production systems, administrative processes as well as in fuel transport, where the transportation itself uses up fuel, but the content of the truck or tanker is emptied at the final destination. In Sect. 3 we discuss another application area in some more detail, viz. biological cell transport.

The three models under consideration in this paper are successively treated in Sects. 2, 3 and 4. The first is a serial model for which the steady-state LaplaceStieltjes transform (LST) of the total workload in the first $k$ sites is derived just after gate openings and at arbitrary epochs. The second model is an ASIP model consisting of only two sites in series, each with its own gate and a leakage of a fixed rate from $Q_{1}$. Gate openings occur at exponentially distributed intervals, and the external input processes to the two sites are nondecreasing Lévy processes. The steady-state joint workload distribution functions right after gate openings, right before gate openings and at arbitrary epochs are derived. The third model is a shot-noise counterpart of the second model where the leakage rate from the first queue is linear in the workload and thus, in between gate openings, behaves like a shot-noise process. We obtain the steady-state joint workload LST just before and just after gate openings.

\section{Model 1: $n$ queues in series}

This section is devoted to an ASIP model consisting of $n$ queues in series. The model is described in Sect. 2.1. In Sect. 2.2 we derive an explicit expression for the steady-state Laplace-Stieltjes transform (LST) of the total workload in the first $k$ queues, just after a gate opening. The workload LST in the first $k$ queues at arbitrary epochs is derived in Sect. 2.3. The steady-state joint workload LST right after gate openings is harder to obtain. In Sect. 2.4 we provide a fairly detailed procedure for obtaining it in the cases $n=2,3$.

\subsection{Model description}

Consider the following model of $n$ queues $Q_{1}, \ldots, Q_{n}$ in series: Each queue has one gate behind it, which may be viewed as a server. Gates are closed almost all the time. When gate $i=1,2, \ldots, n-1$ (the gate behind $Q_{i}$ ) opens, all the work present in $Q_{i}$ is instantaneously transferred to $Q_{i+1}$. When gate $n$ opens, all the work present in $Q_{n}$ instantaneously leaves the system. After the transfer, the gate immediately closes again. Gate openings are determined by a Markov renewal process. If, at some time $t$, gate $i$ opens, then with probability $p_{i j}$ the next gate to open is gate $j$ and the time until that gate opens is an independent random variable distributed like $O_{i j}$. We assume 
that the Markov chain governing the successive gate openings is irreducible and we denote its steady-state distribution by $\pi_{i}, i=1, \ldots, n$.

During an $O_{i j}$ period, work (sometimes denoted as fluid) may externally arrive at all queues. The LST of amounts of work arriving to $Q_{1}, \ldots, Q_{n}$ during an $O_{i j}$ period is given by $A_{i j}\left(s_{1}, \ldots, s_{n}\right)$. Given $O_{i j}$, these amounts are independent of amounts arriving during previous periods. In addition, we denote the LST of the cumulative amount of work arriving to $Q_{1}, \ldots, Q_{k}$ during an $O_{i j}$ period by $A_{i j k}(s)=A_{i j}(s, \ldots, s, 1, \ldots, 1)$, where the last $s$ occurs at position $k$. Notice that one example is provided by an $n$-dimensional Lévy subordinator process, possibly with dependence between amounts arriving at different queues and with Laplace exponents which may depend on the type of gate opening interval.

We recall that we restrict ourselves to the case in which work from $Q_{i}$ can only move to $Q_{i+1}, i=1,2, \ldots, n-1$. That assumption will allow us to obtain exact steady-state results for the total amount of work $V_{(k)}$ which is present in the first $k$ queues right after a gate opening $(k=1,2, \ldots, n)$. Our results will become somewhat simpler in the special case in which the next gate opening is of gate $j$ with a fixed probability $q_{j}$, i.e., irrespective of the index of the previous gate opening.

\subsection{Analysis of the total workload in the first $k$ queues}

We are interested in the steady-state joint distribution of the amounts of work $\left(V_{1}, \ldots, V_{n}\right)$ just after a gate opening. To argue the existence of such a distribution, one can follow a similar reasoning as in Section 2 of [6], which also considers an ASIP model of $n$ queues in series, but in which the focus is on customers instead of work/fluid.

In the present subsection we shall in particular focus on $V_{(k)}=V_{1}+\cdots+V_{k}$, namely the total amount of work in the first $k$ queues right after a gate opening. It will turn out that the analysis of $V_{(k)}$ can closely follow the reasoning for queue lengths in [6].

Introducing $M$, the index of the gate that has just opened, we consider

$$
\xi_{k i}(s)=\mathbb{E}\left[\mathrm{e}^{-s V_{(k)}} 1_{\{M=i\}}\right], \quad k, i=1, \ldots, n,
$$

where $1_{\{\cdot\}}$ denotes an indicator. The fact that fluid can only move to downstream queues (i.e., with higher index) will allow us to express all $\xi_{k i}(s)$ for a fixed $k$ as functions of $\xi_{k-1, j}(s)$ and, inductively, as functions of $\xi_{1 j}(s)$, which can be determined explicitly.

Step 1: Determination of $\xi_{1 j}(s), j=1, \ldots, n$.

Obviously

$$
\xi_{11}(s)=\mathbb{P}(M=1)=\pi_{1} .
$$

Indeed, after gate 1 has opened, $Q_{1}$ instantaneously has become empty. Now consider two successive gate openings in steady state, the latter one being an opening of gate 
$j$. Summing over all possible gates $i$ opened at the previous gate opening gives

$$
\xi_{1 j}(s)=\sum_{i=1}^{n} \xi_{1 i}(s) p_{i j} A_{i j 1}(s)=\sum_{i=2}^{n} \xi_{1 i}(s) p_{i j} A_{i j 1}(s)+\xi_{11}(s) p_{1 j} A_{1 j 1}(s), \quad j \neq 1
$$

Here we have employed $A_{i j 1}(s)$, the LST of the amount of work arriving at $Q_{1}$ during the gate opening interval.

Introducing the $(n-1)$-dimensional vectors

$$
\xi_{1}(s)=\left(\xi_{12}(s), \ldots, \xi_{1 n}(s)\right), \quad R_{1}(s)=\left(p_{12} A_{121}(s), \ldots, p_{1 n} A_{1 n 1}(s)\right)
$$

and with the $(n-1) \times(n-1)$ matrix $P_{1}(s)$ of which the $(i-1, j-1)$ th coordinate is $p_{i j} A_{i j 1}(s), i, j=2, \ldots, n$, we can write (3) as

$$
\xi_{1}(s)=\xi_{1}(s) P_{1}(s)+\xi_{11}(s) R_{1}(s)
$$

and hence, with $I$ the matrix with ones on the diagonal and zeroes outside the diagonal,

$$
\xi_{1}(s)=\xi_{11}(s) R_{1}(s)\left(I-P_{1}(s)\right)^{-1}
$$

All the terms in the right-hand side of (5) are known; in particular, $\xi_{11}(s)=\pi_{1}$ is given in (2). Hence, we have determined $\xi_{11}(s), \xi_{12}(s), \ldots, \xi_{1 n}(s)$.

Step 2: Expressing $\xi_{k j}(s)$ in terms of $\xi_{k-1, i}(s)$, for $i, j=1, \ldots, n, k=2, \ldots, n$.

Considering two successive gate openings in steady state, the last one being of gate $j$, and summing over all possible gates $i$ for the first gate opening, we have for $k=$ $2, \ldots, n$,

$$
\xi_{k j}(s)=\sum_{i=1}^{n} \xi_{k i}(s) p_{i j} A_{i j k}(s)=\sum_{i \neq k} \xi_{k i}(s) p_{i j} A_{i j k}(s)+\xi_{k k}(s) p_{k j} A_{k j k}(s), \quad j \neq k,
$$

whereas

$$
\xi_{k k}(s)=\sum_{i=1}^{n} \xi_{k-1, i}(s) p_{i k} A_{i k, k-1}(s) .
$$

The explanation for the deviating terms $\left(\xi_{k-1, i}(s)\right.$ instead of $\xi_{k i}(s)$ and $A_{i k, k-1}(s)$ instead of $\left.A_{i k k}(s)\right)$ is that $Q_{k}$ has become empty right after an opening of gate $k$, so that the total amount of fluid present in $Q_{1}, \ldots, Q_{k}$ equals the total amount present in $Q_{1}, \ldots, Q_{k-1}$ after the previous gate opening, plus the amount of fluid arriving in the first $k-1$ queues.

Introducing the $(n-1)$-dimensional vectors

$$
\begin{aligned}
\xi_{k}(s) & =\left(\xi_{k 1}(s), \ldots, \xi_{k, k-1}(s), \xi_{k, k+1}(s), \ldots, \xi_{k n}(s)\right), \\
R_{k}(s) & =\left(p_{k 1} A_{k 1 k}(s), \ldots, p_{k, k-1} A_{k, k-1, k}(s), p_{k, k+1} A_{k, k+1, k}(s), \ldots, p_{k n} A_{k n k}(s)\right),
\end{aligned}
$$


and with the matrix $P_{k}(s)=\left(p_{i j} A_{i j k}(s)\right)_{i, j \neq k}$ we can write (6) as

$$
\xi_{k}(s)=\xi_{k}(s) P_{k}(s)+\xi_{k k}(s) R_{k}(s)
$$

yielding

$$
\xi_{k}(s)=\xi_{k k}(s) R_{k}(s)\left(I-P_{k}(s)\right)^{-1}
$$

Introducing

$$
\begin{aligned}
& C_{k-1}(s)=\left(p_{1 k} A_{1 k, k-1}(s), \ldots, p_{k-2, k} A_{k-2, k, k-1}(s),\right. \\
& \left.p_{k k} A_{k k, k-1}(s), \ldots, p_{n k} A_{n k, k-1}(s)\right)
\end{aligned}
$$

we can rewrite (7) as

$$
\xi_{k k}(s)=\xi_{k-1}(s) C_{k-1}^{T}(s)+\xi_{k-1, k-1}(s) p_{k-1, k} A_{k-1, k, k-1}(s) .
$$

We have thus expressed $\xi_{k}(s)$ in terms of $\xi_{k k}(s)$ via (9), and $\xi_{k k}(s)$ in terms of $\xi_{k-1}(s)$ and $\xi_{k-1, k-1}(s)$ via (10). Iterating, defining an empty product to be one and defining $\xi_{0}(s) C_{0}^{T}(s)$ to equal $\pi_{1}$ for notational convenience, we obtain

$$
\xi_{k k}(s)=\sum_{i=0}^{k-1} \xi_{i}(s) C_{i}^{T}(s) \prod_{j=i+1}^{k-1} p_{j, j+1} A_{j, j+1, j}(s)
$$

By carefully studying the structure of the above recursions, and introducing

$$
H_{i}(s)=R_{i}(s)\left(I-P_{i}(s)\right)^{-1} C_{i}^{T}(s), \quad i=1, \ldots, n,
$$

the following holds:

$$
\xi_{k k}(s)=\pi_{1} \sum_{\ell_{1}, \ldots, \ell_{k-1} \in\{0,1\}} \prod_{i=1}^{k-1}\left(\ell_{i} H_{i}(s)+\left(1-\ell_{i}\right) p_{i, i+1} A_{i, i+1, i}(s)\right), \quad k=1, \ldots, n
$$

With (12) and (9) we have a recipe for determining $\xi_{k j}(s)$ explicitly, for $k, j=$ $1, \ldots, n$.

Example Let us consider the special case in which $p_{i j}=p_{1 j}, \forall i, j$, and $A_{i j k}(s)=$ $A_{1 j k}(s), \forall i, j, k$. Namely, the Markov renewal process that determines the gate openings and the intervals in between has a simple structure. Each time the next gate opening is of gate $j$ with probability $p_{1 j}$, and the interval length until the next opening also only depends on $j$. In this case we can obtain a simple expression for $\mathbb{E}\left[\mathrm{e}^{-s V_{(k)}}\right]=\sum_{j=1}^{n} \xi_{k j}(s)$. We have

$$
\xi_{11}(s)=\pi_{1}=p_{11},
$$


and, summing (3) over $j=2, \ldots, n$,

$$
\mathbb{E}\left[\mathrm{e}^{-s V_{(1)}}\right]=\sum_{j=1}^{n} \xi_{1 j}(s)=p_{11}+\sum_{j=2}^{n} p_{1 j} A_{1 j 1}(s) \mathbb{E}\left[\mathrm{e}^{-s V_{(1)}}\right],
$$

yielding

$$
\mathbb{E}\left[\mathrm{e}^{-s V_{(1)}}\right]=\frac{p_{11}}{1-\sum_{j=2}^{n} p_{1 j} A_{1 j 1}(s)} .
$$

Furthermore, summing (6) over $j \neq k$ and using (7),

$$
\mathbb{E}\left[\mathrm{e}^{-s V_{(k)}}\right]=p_{1 k} A_{1 k, k-1}(s) \mathbb{E}\left[\mathrm{e}^{-s V_{(k-1)}}\right]+\sum_{j \neq k} p_{1 j} A_{1 j k}(s) \mathbb{E}\left[\mathrm{e}^{-s V_{(k)}}\right],
$$

leading to the following recursive expression of $\mathbb{E}\left[\mathrm{e}^{-s V_{(k)}}\right]$ in terms of $\mathbb{E}\left[\mathrm{e}^{-s V_{(k-1)}}\right]$ :

$$
\mathbb{E}\left[\mathrm{e}^{-s V_{(k)}}\right]=\frac{p_{1 k} A_{1 k, k-1}(s)}{1-\sum_{j \neq k} p_{1 j} A_{1 j k}(s)} \mathbb{E}\left[\mathrm{e}^{-s V_{(k-1)}}\right] .
$$

Via iteration we obtain

$$
\mathbb{E}\left[\mathrm{e}^{-s V_{(k)}}\right]=\prod_{i=1}^{k} \frac{p_{1 i} A_{1 i, i-1}(s)}{1-\sum_{j \neq i} p_{1 j} A_{1 j k}(s)},
$$

where $A_{110}(s)=1$.

Formula (18) reveals a decomposition property. That is, the LST is a product of $k$ terms, all of which are LSTs of random variables, and this implies that $V_{(k)}$ can be represented as sum of $k$ independent random variables.

\subsection{The workload distribution at an arbitrary epoch}

Armed with the LSTs $\xi_{k i}(s)$ from the previous subsection, we shall now derive an expression for the steady-state LST $\chi_{k}(s)$ of the total workload in the first $k$ queues at an arbitrary epoch. In order to do this, we need to further specify the arrival process. Indeed, it clearly makes a difference whether the amounts of work which arrive in the queues during a gate opening interval $O_{i j}$ enter the system at the beginning of such an interval, or at the end, or according to some other stochastic process. In this subsection we shall assume that the external arrival process is an $n$ dimensional subordinator (hence a nondecreasing Lévy process) which may vary from one gate interval to another. Denote the Lévy input process during an $O_{i j}$ period by $\left\{X_{i j}^{(1)}(t), \ldots, X_{i j}^{(n)}(t), t \geq 0\right\}$ and its Laplace exponent by $-\eta_{i j}\left(s_{1}, \ldots, s_{n}\right)$, i.e.,

$$
\mathbb{E}\left[\mathrm{e}^{-s_{1} X_{i j}^{(1)}(t)-\cdots-s_{n} X_{i j}^{(n)}(t)}\right]=\mathrm{e}^{-t \eta_{i j}\left(s_{1}, \ldots, s_{n}\right)} .
$$


The LST $\chi_{k}(s)$ is obtained by averaging over all possible gate intervals, and by observing that the amount of work present in the first $k$ queues at an arbitrary epoch in an $O_{i j}$ interval is the sum of the amount of work just after the corresponding gate $i$ opening (which has LST $\xi_{k i}(s)$ ), and-independent of it - the amount of work $\zeta_{i j}$ that has arrived in the past part of that interval:

$$
\chi_{k}(s)=\frac{\sum_{i} \sum_{j} \pi_{i} p_{i j} \mathbb{E}\left[O_{i j}\right] \xi_{k i}(s) \mathbb{E}\left[\mathrm{e}^{-s \zeta_{i j}}\right]}{\sum_{i} \sum_{j} \pi_{i} p_{i j} \mathbb{E}\left[O_{i j}\right]}
$$

where $\xi_{k i}(s), k \neq i$, are given in (9) and $\xi_{k k}(s)$ in (12). Considering an $O_{i j}$ interval at an arbitrary epoch during that interval, the LST of the work $\zeta_{i j}$ that has arrived at the queues during $O_{i j}^{\text {past }}$, the past part of $O_{i j}$, equals (using that $O_{i j}^{\text {past }}$ has density $\frac{\mathbb{P}\left(O_{i j}>t\right)}{\mathbb{E}\left[O_{i j}\right]}$ and denoting the LST of $O_{i j}$ by $\left.\omega_{i j}(s)\right)$

$$
\begin{aligned}
\int_{0}^{\infty} \mathbb{E}\left[\mathrm{e}^{\left.-s \sum_{m=1}^{k} X_{i j}^{(m)}(t)\right]} \frac{\mathbb{P}\left(O_{i j}>t\right)}{\mathbb{E}\left[O_{i j}\right]} \mathrm{d} t\right. & =\int_{0}^{\infty} \mathrm{e}^{-t \eta_{i j}(s, \ldots, s, 0, \ldots, 0)} \frac{\mathbb{P}\left(O_{i j}>t\right)}{\mathbb{E}\left[O_{i j}\right]} \mathrm{d} t \\
& =\frac{1-\omega_{i j}\left(\eta_{i j}(s, \ldots, s, 0, \ldots, 0)\right)}{\mathbb{E}\left[O_{i j}\right] \eta_{i j}(s, \ldots, s, 0, \ldots, 0)}
\end{aligned}
$$

where the last $s$ in the $n$-dimensional expressions in the above formula occurs at position $k$. Hence,

$$
\chi_{k}(s)=\frac{\sum_{i} \sum_{j} \pi_{i} p_{i j} \xi_{k i}(s) \frac{1-\omega_{i j}\left(\eta_{i j}(s, \ldots, s, 0, \ldots, 0)\right)}{\eta_{i j}(s, \ldots, s, 0, \ldots, 0)}}{\sum_{i} \sum_{j} \pi_{i} p_{i j} \mathbb{E}\left[O_{i j}\right]}
$$

When $O_{i j} \sim \exp \left(\lambda_{i j}\right),(20)$ becomes

$$
\chi_{k}(s)=\frac{\sum_{i} \sum_{j} \frac{\pi_{i} p_{i j}}{\lambda_{i j}+\eta_{i j}(s, \ldots, s, 0, \ldots, 0)} \xi_{k i}(s)}{\sum_{i} \sum_{j} \frac{\pi_{i} p_{i j}}{\lambda_{i j}}}
$$

\subsection{Multi-dimensional workload distributions}

In this subsection we outline how the joint workload distribution just after gate openings can be obtained. We provide a fairly detailed procedure for the cases $n=2$ and $n=3$ and, for the sake of brevity, under the simplifying assumptions that $p_{i j}=q_{j}$ for all relevant $i$ and that $A_{i j}(\cdot)=A(\cdot)$ for all relevant $i, j$. For higher values of $n$, as well as without these simplifying assumptions, a similar procedure can be followed; however, it leads to quite messy expressions.

The case $n=2$

We shall determine the LST of the steady-state joint distribution of the workloads right after gate openings, $\xi\left(s_{1}, s_{2}\right)=\mathbb{E}\left[\mathrm{e}^{-s_{1} V_{1}-s_{2} V_{2}}\right]$. 
If $V_{i}^{(r)}$ denotes the amount of work in $Q_{i}$ immediately after the $r$ th gate opening, and $A_{i}^{(r+1)}$ the amount of fluid entering $Q_{i}$ between the $r$ th and $(r+1)$ st gate openings, then

$$
V_{1}^{(r+1)}=0, \quad V_{2}^{(r+1)}=V_{1}^{(r)}+A_{1}^{(r+1)}+V_{2}^{(r)}+A_{2}^{(r+1)},
$$

if the $(r+1)$ st gate opening is of gate 1 , and

$$
V_{1}^{(r+1)}=V_{1}^{(r)}+A_{1}^{(r+1)}, \quad V_{2}^{(r+1)}=0,
$$

if the $(r+1)$ st gate opening is of gate 2 . In steady state this yields

$$
\xi\left(s_{1}, s_{2}\right)=q_{1} A\left(s_{2}, s_{2}\right) \xi\left(s_{2}, s_{2}\right)+q_{2} A\left(s_{1}, 0\right) \xi\left(s_{1}, 0\right) .
$$

Now observe that $\xi\left(s_{1}, 0\right)=\xi_{1}\left(s_{1}\right)$, and that this term, which only refers to $Q_{1}$, can be obtained from the results of Sect. 2.2. Furthermore, observe that $\xi\left(s_{2}, s_{2}\right)=$ $\mathbb{E}\left[\mathrm{e}^{-s_{2} V^{(2)}}\right]$, a result for the total workload in $Q_{1}+Q_{2}$, which also follows from Sect. 2.2. We are thus able to obtain $\xi\left(s_{1}, s_{2}\right)$.

Remark 1 Let us assume that station 2 is replaced by $L$ parallel stations $Q_{21}, \ldots, Q_{2 L}$. A proportion $p_{j}$ of every drop that leaves station $Q_{1}$ is routed to station $Q_{2 j}$ and the gates at station 2 open at the same times. Let $1-\sum_{j=1}^{L} p_{j}$ be the proportion that leave the system entirely (from station $Q_{1}$ ). If we denote by $V_{1}, V_{21}, \ldots, V_{2 L}$ the workloads in all stations, then it is easily seen that $V_{2 j}=p_{j} V_{2}$, where $\left(V_{1}, V_{2}\right)$ was defined at the beginning of this subsection. This immediately implies that the steady-state LST for this case becomes

$$
\mathbb{E}\left[\mathrm{e}^{-\alpha_{1} V_{1}-\sum_{j=1}^{L} \alpha_{2 j} V_{2 j}}\right]=\mathbb{E}\left[\mathrm{e}^{-\alpha_{1} V_{1}-\sum_{j=1}^{L} \alpha_{2 j} p_{j} V_{2}}\right]=\xi\left(\alpha_{1}, \sum_{j=1}^{L} p_{j} \alpha_{2 j}\right)
$$

This remains true regardless of the simplifying assumptions or the assumptions on the arrival process and, in fact, in the $n$ station case, every station can be replaced by parallel stations in a similar manner with the same consequence.

The case $n=3$

Here we compute the three-dimensional steady-state transform $\xi\left(s_{1}, s_{2}, s_{3}\right)=$ $\mathbb{E}\left[\mathrm{e}^{-s_{1} V_{1}-s_{2} V_{2}-s_{3} V_{3}}\right]$ of workload right after gate openings (under the same simplifying assumptions described in the beginning of this subsection). We have

$$
V_{1}^{(r+1)}=0, \quad V_{2}^{(r+1)}=V_{1}^{(r)}+A_{1}^{(r+1)}+V_{2}^{(r)}+A_{2}^{(r+1)}, \quad V_{3}^{(r+1)}=V_{3}^{(r)}+A_{3}^{(r+1)},
$$

if the $(r+1)$ st gate opening is of gate 1 , and

$$
V_{1}^{(r+1)}=V_{1}^{(r)}+A_{1}^{(r+1)}, \quad V_{2}^{(r+1)}=0, \quad V_{3}^{(r+1)}=V_{2}^{(r)}+A_{2}^{(r+1)}+V_{3}^{(r)}+A_{3}^{(r+1)},
$$


if the $(r+1)$ st gate opening is of gate 2 , and

$$
V_{1}^{(r+1)}=V_{1}^{(r)}+A_{1}^{(r+1)}, \quad V_{2}^{(r+1)}=V_{2}^{(r)}+A_{2}^{(r+1)}, \quad V_{3}^{(r+1)}=0,
$$

if the $(r+1)$ st gate opening is of gate 3 . Here the $A^{(r+1)}$ terms are independent of the $V^{(r)}$ terms. In steady state, this yields

$$
\begin{aligned}
\xi\left(s_{1}, s_{2}, s_{3}\right)= & q_{1} A\left(s_{2}, s_{2}, s_{3}\right) \xi\left(s_{2}, s_{2}, s_{3}\right)+q_{2} A\left(s_{1}, s_{3}, s_{3}\right) \xi\left(s_{1}, s_{3}, s_{3}\right) \\
& +q_{3} A\left(s_{1}, s_{2}, 0\right) \xi\left(s_{1}, s_{2}, 0\right) .
\end{aligned}
$$

Taking $s_{3}=0$ gives

$$
\xi\left(s_{1}, s_{2}, 0\right)=\frac{q_{1} A\left(s_{2}, s_{2}, 0\right) \xi\left(s_{2}, s_{2}, 0\right)+q_{2} A\left(s_{1}, 0,0\right) \xi\left(s_{1}, 0,0\right)}{1-q_{3} A\left(s_{1}, s_{2}, 0\right)} .
$$

Notice that $\xi\left(s_{1}, 0,0\right)=\mathbb{E}\left[\mathrm{e}^{-s_{1} V^{(1)}}\right]$ and that $\xi\left(s_{2}, s_{2}, 0\right)=\mathbb{E}\left[\mathrm{e}^{-s_{2} V^{(2)}}\right]$ are known from the previous section, so that $\xi\left(s_{1}, s_{2}, 0\right)$ is known. Of course, this term is also closely related to the result in (22) for a model with $n=2$ queues. In fact, a straightforward extension of (22) for the first two queues of an $n$-queue tandem ASIP is

$$
\begin{aligned}
\xi\left(s_{1}, s_{2}, 0, \ldots, 0\right)= & q_{1} A\left(s_{2}, s_{2}, 0, \ldots, 0\right) \xi\left(s_{2}, s_{2}, 0, \ldots, 0\right) \\
& +q_{2} A\left(s_{1}, 0,0, \ldots, 0\right) \xi\left(s_{1}, 0,0, \ldots, 0\right) \\
& +\sum_{j=3}^{n} q_{j} A\left(s_{1}, s_{2}, 0, \ldots, 0\right) \xi\left(s_{1}, s_{2}, 0, \ldots, 0\right) .
\end{aligned}
$$

We still need to determine $\xi\left(s_{2}, s_{2}, s_{3}\right)$ and $\xi\left(s_{1}, s_{3}, s_{3}\right)$ in (24). Take $s_{2}=s_{3}$ in (24) to get

$$
\begin{aligned}
\xi\left(s_{1}, s_{3}, s_{3}\right)= & q_{1} A\left(s_{3}, s_{3}, s_{3}\right) \xi\left(s_{3}, s_{3}, s_{3}\right)+q_{2} A\left(s_{1}, s_{3}, s_{3}\right) \xi\left(s_{1}, s_{3}, s_{3}\right) \\
& +q_{3} A\left(s_{1}, s_{3}, 0\right) \xi\left(s_{1}, s_{3}, 0\right) .
\end{aligned}
$$

This equation allows us to express $\xi\left(s_{1}, s_{3}, s_{3}\right)$ in terms of the, by now known, functions $\xi\left(s_{3}, s_{3}, s_{3}\right)=\mathbb{E}\left[\mathrm{e}^{-s_{3} V^{(3)}}\right]$ and $\xi\left(s_{1}, s_{3}, 0\right)$ (cf. (25)). It remains to determine $\xi\left(s_{2}, s_{2}, s_{3}\right)$. For this purpose, take $s_{1}=s_{2}$ in (24):

$$
\begin{aligned}
\xi\left(s_{2}, s_{2}, s_{3}\right)= & q_{1} A\left(s_{2}, s_{2}, s_{3}\right) \xi\left(s_{2}, s_{2}, s_{3}\right)+q_{2} A\left(s_{2}, s_{3}, s_{3}\right) \xi\left(s_{2}, s_{3}, s_{3}\right) \\
& +q_{3} A\left(s_{2}, s_{2}, 0\right) \xi\left(s_{2}, s_{2}, 0\right) .
\end{aligned}
$$

This equation allows us to express $\xi\left(s_{2}, s_{2}, s_{3}\right)$ in terms of the, by now known, functions $\xi\left(s_{2}, s_{3}, s_{3}\right)$ and $\xi\left(s_{2}, s_{2}, 0\right)=\mathbb{E}\left[\mathrm{e}^{-s_{2} V^{(2)}}\right]$. Thus, $\xi\left(s_{1}, s_{2}, s_{3}\right)$ has been obtained. 


\section{Model 2: an ASIP with leakage}

In this section we consider an ASIP consisting of two stations $Q_{1}$ and $Q_{2}$ in series, each with its own gate, with the additional feature that there is leakage from $Q_{1}$. Namely, the content of $Q_{1}$ is not only transferred to $Q_{2}$ at openings of the gate after $Q_{1}$, but the content also leaks at a fixed rate out of $Q_{1}$ (whenever the queue is not empty). We restrict ourselves in this section to gate openings at i.i.d. exponentially distributed intervals, and we assume that the external input processes to the two stations are two independent subordinators (nondecreasing Lévy processes). In Sect. 3.1 we present some preliminary results on a Lévy process reflected at zero, which are used in Sect. 3.2 to derive the steady-state joint workload distribution right after gate openings, right before gate openings and at arbitrary epochs.

In Sect. 1 we provided some motivation for introducing the feature of leakage in ASIP models. Here we would like to mention one specific application area in biology in which such a combination of gate openings and leakage appears quite naturally: cellular transport. Cells in the human body require a large variety of particles to sustain themselves. As explained in Sections 2 and 3 of [13], a cell wall is semipermeable: Particles like water, carbon dioxide and oxygen can cross it. They get molecules from a nearby blood vessel (arrivals). Inside a cell, a particle is consumed (energy; this corresponds to leakage out of the system). Single particles can cross a cell wall by using energy from an ATP, i.e., adenosine triphosphate, molecule (leakage from one cell to the next one). In addition, a vesicle captures particles and moves toward the cell wall, allowing the particles to cross it $[3,13]$. Such vesicle-mediated transport is in our model represented by the gate openings.

\subsection{Preliminaries}

Let $X=\{X(t) \mid t \geq 0\}$ be a Lévy process with no negative jumps which is not a subordinator and with Laplace exponent $\varphi(\alpha)=\log \mathbb{E}\left[\mathrm{e}^{-\alpha X(1)}\right]$. Denote

$$
\begin{aligned}
& L_{x}(t)=-\inf _{0 \leq s \leq t}(x+X(s))^{-}=\left(L_{0}(t)-x\right)^{+}, \\
& Z_{x}(t)=x+X(t)+L_{x}(t)=X(t)+x \vee L_{0}(t),
\end{aligned}
$$

and finally, for $u \geq 0$ let

$$
\psi(u)=\inf \{\alpha \mid \varphi(\alpha)>u\} .
$$

Assuming that $T \sim \exp (\lambda)$ is independent of $X$, then for any $\alpha \geq 0$, and $\beta>$ $-\psi(\lambda)$ we have that

$$
\mathbb{E}\left[\mathrm{e}^{-\alpha Z_{x}(T)-\beta L_{x}(T)}\right]=\frac{e^{-\alpha x}(\psi(\lambda)+\beta)-\mathrm{e}^{-\psi(\lambda) x}(\alpha+\beta)}{\left(1-\frac{\varphi(\alpha)}{\lambda}\right)(\psi(\lambda)+\beta)},
$$

where for $\alpha=\psi(\lambda)$ the right-hand side is defined by continuity via L'Hôpital's rule. This is, in essence, Theorem 3.10 on page 259 of [1], which is a direct application 
of [14]. It is easy to check that the proof is valid for all $\alpha, \beta$ as given here and not just $\alpha, \beta>0$ as in [1]. This will be important later.

Whenever $Y=\{Y(t) \mid t \geq 0\}$ is a measurable process which is independent of $T_{\lambda} \sim \exp (\lambda)$, then clearly, for every $\gamma>-\lambda$ we have that

$$
\mathbb{E}\left[Y\left(T_{\lambda}\right) e^{-\gamma T_{\lambda}}\right]=\int_{0}^{\infty} \mathbb{E}[Y(t)] \mathrm{e}^{-\gamma t} \lambda \mathrm{e}^{-\lambda t} \mathrm{~d} t=\frac{\lambda}{\lambda+\gamma} \mathbb{E}\left[Y\left(T_{\lambda+\gamma}\right)\right] .
$$

From (32) and (33) it immediately follows that, for each $\alpha \geq 0, \gamma>-\lambda$ and $\beta>$ $-\psi(\lambda+\gamma)$

$$
\begin{aligned}
\mathbb{E}\left[\mathrm{e}^{-\alpha Z_{x}(T)-\beta L_{x}(T)-\gamma T}\right] & =\frac{\lambda}{\lambda+\gamma} \cdot \frac{\mathrm{e}^{-\alpha x}(\psi(\lambda+\gamma)+\beta)-\mathrm{e}^{-\psi(\lambda+\gamma) x}(\alpha+\beta)}{\left(1-\frac{\varphi(\alpha)}{\lambda+\gamma}\right)(\psi(\lambda+\gamma)+\beta)} \\
& =\frac{\mathrm{e}^{-\alpha x}(\psi(\lambda+\gamma)+\beta)-\mathrm{e}^{-\psi(\lambda+\gamma) x}(\alpha+\beta)}{\left(1+\frac{\gamma-\varphi(\alpha)}{\lambda}\right)(\psi(\lambda+\gamma)+\beta)}
\end{aligned}
$$

\subsection{Analysis}

Now consider a system consisting of two stations in series. The external input process of station $Q_{i}$ is a nondecreasing Lévy process $J_{i}, i=1,2$. These are independent subordinators with

$$
\eta_{i}(\alpha)=-\log \mathrm{e}^{-\alpha J_{i}(1)}=c_{i} \alpha+\int_{(0, \infty)}\left(1-\mathrm{e}^{-\alpha u}\right) \nu_{i}(\mathrm{~d} u),
$$

where $c_{i} \geq 0$ and $v_{i}$ is a Lévy measure satisfying $\int_{(0, \infty)}(u \wedge 1) v_{i}(\mathrm{~d} u)<\infty$ and $v_{i}(-\infty, 0]=0$.

The cumulative input to $Q_{1}$ is $x_{1}+J_{1}(t)$, where $x_{1} \geq 0$ is its initial state. Whenever $Q_{1}$ is not empty, the content leaks (is processed) at some rate $r \geq 0$. When $c_{1}<r$ and $Q_{1}$ is empty, the leak is at rate $c_{1}$. A proportion $p \in[0,1]$ leaks into $Q_{2}$ and the rest leaves the system altogether. At independent intervals, also independent of $J_{1}, J_{2}$ and distributed as $\exp \left(\lambda_{1}\right)$, the entire content of $Q_{1}$ is transferred to $Q_{2}$. As for $Q_{2}$, the cumulative input is whatever arrives from $Q_{1}$ (either from the leakage or from the occasional transfer) as well as $x_{2}+J_{2}(t)$, where $x_{2} \geq 0$ is the initial state of $Q_{2}$. At independent intervals which are distributed as $\exp \left(\lambda_{2}\right)$ and independent of everything else (including the inter-transfer times of $Q_{1}$ ) all the available content of $Q_{2}$ leaves the system all at once. This is the two-queue ASIP system that we would like to explore.

Denote $\lambda=\lambda_{1}+\lambda_{2}$. For $X_{1}(t)=J_{1}(t)-r t$, with Laplace-Stieltjes exponent $\varphi_{1}(\alpha)=\log \mathbb{E}\left[\mathrm{e}^{-\alpha X_{1}(1)}\right]=r \alpha-\eta_{1}(\alpha)$, the content of $Q_{1}$ at time $t \geq 0$ is $Z_{1, x_{1}}(t)$, where $Z_{1, x_{1}}$ replaces $Z_{x}$ in (30). As long as there is no transfer until time $t \geq 0$, the input to $Q_{2}$ is $p\left(r t-L_{1, x_{1}}(t)\right)$, as $L_{1, x_{1}}(t)$ is the cumulative lost capacity.

If $T \sim \exp (\lambda)$ (the minimum of the transfer times from $Q_{1}$ and $Q_{2}$ ), then with probability $\lambda_{1} / \lambda$ there is a transfer from $Q_{1}$ to $Q_{2}$, in which case the state of the stations will be $\left(0, Z_{1, x_{1}}(T)+p\left(r T-L_{1, x_{1}}(T)\right)+x_{2}+J_{2}(T)\right)$, and with probability 
$\lambda_{2} / \lambda$ the state will be $\left(Z_{1, x_{1}}(T), 0\right)$. Therefore, we will be interested in the LST of $Z_{1, x_{1}}(T)+p\left(r T-L_{1, x_{1}}(T)\right)+J_{2}(T)$ and that of $Z_{1, x_{1}}(T)$.

If $r \leq c_{1}$ (which includes the case $r=0$ ), then $X_{1}$ is a subordinator and (34) does not apply. However, in this case the result is far simpler, as $L_{1, x_{1}}(T)=0$ and $Z_{1, x_{1}}(T)=x_{1}+X_{1}(T)$. When $r>c_{1}$ then, noting that

$$
\mathbb{E}\left[\mathrm{e}^{-\alpha\left(Z_{1, x_{1}}(T)+p\left(r T-L_{1, x_{1}}(T)\right)+J_{2}(T)\right)}\right]=\mathbb{E}\left[\mathrm{e}^{-\left(\alpha Z_{1, x_{1}}(T)-\alpha p L_{1, x_{1}}(T)+\left(\operatorname{pr} \alpha+\eta_{2}(\alpha)\right) T\right)}\right],
$$

we simply apply (34), setting either $\gamma=p r \alpha+\eta_{2}(\alpha)$ and $\beta=-p \alpha$ (for $Z_{1, x_{1}}(T)+$ $\left.p\left(r T-L_{1, x_{1}}(T)\right)+J_{2}(T)\right)$ or $\gamma=\beta=0$ (for $Z_{1, x_{1}}(T)$ ). Recall that in order to use (34) we must have $\beta>-\psi_{1}(\lambda+\gamma)$, cf. (31). To see that this holds in this case, note that, when $\beta<0$, this is equivalent to $\varphi_{1}(-\beta)<\lambda+\gamma$ and if we insert $\beta=-p \alpha$ and $\gamma=\operatorname{pr} \alpha+\eta_{2}(\alpha)$, and observe that $\varphi_{1}(\alpha)=r \alpha-\eta_{1}(\alpha)$, then indeed, as required,

$$
\varphi_{1}(-\beta)=r p \alpha-\eta_{1}(p \alpha)<\lambda+\operatorname{pr} \alpha+\eta_{2}(\alpha)=\lambda+\gamma
$$

This system is regenerative. The reason is that starting from $\left(x_{1}, x_{2}\right)$ the system will (almost surely) reach some state $\left(0, x_{2}^{\prime}\right)$ after which it will reach a state $\left(Z_{1,0}(\tau), 0\right)$, where $\tau$ is the first time thereafter that the second station is emptied. This state is a regenerative one, and it is clear that the inter-regeneration times distribution has a finite mean and is nonarithmetic; it actually has a density. Thus, there exists a limiting=ergodic=stationary distribution for the joint content process. Assume that $\left(Z_{1}, Z_{2}\right)$ has this joint distribution (of the buffer contents right after an arbitrary gate opening) and denote $f_{A}\left(\alpha_{1}, \alpha_{2}\right)=\mathbb{E}\left[\mathrm{e}^{-\alpha_{1} Z_{1}-\alpha_{2} Z_{2}}\right]$. Then, given the preceding arguments, we must have for the case where $r>c_{1}$ that

$$
\begin{aligned}
& f_{A}\left(\alpha_{1}, \alpha_{2}\right) \\
& =\frac{\lambda_{1}}{\lambda} \frac{f_{A}\left(\alpha_{2}, \alpha_{2}\right)\left(\psi_{1}\left(\lambda+p r \alpha_{2}+\eta_{2}\left(\alpha_{2}\right)\right)-p \alpha_{2}\right)-f_{A}\left(\psi_{1}\left(\lambda+p r \alpha_{2}+\eta_{2}\left(\alpha_{2}\right)\right), \alpha_{2}\right) \alpha_{2}(1-p)}{\left(1-\frac{(1-p) r \alpha_{2}-\eta_{1}\left(\alpha_{2}\right)-\eta_{2}\left(\alpha_{2}\right)}{\lambda}\right)\left(\psi_{1}\left(\lambda+p r \alpha_{2}+\eta_{2}\left(\alpha_{2}\right)\right)-p \alpha_{2}\right)} \\
& \quad+\frac{\lambda_{2}}{\lambda} \frac{f_{A}\left(\alpha_{1}, 0\right) \psi_{1}(\lambda)-f_{A}\left(\psi_{1}(\lambda), 0\right) \alpha_{1}}{\left(1-\frac{r \alpha_{1}-\eta_{1}\left(\alpha_{1}\right)}{\lambda}\right) \psi_{1}(\lambda)} .
\end{aligned}
$$

We shall successively determine (i) $f_{A}\left(\alpha_{1}, 0\right)$ and $f_{A}\left(\psi_{1}(\lambda), 0\right)$, (ii) $f_{A}\left(\alpha_{2}, \alpha_{2}\right)$, $f_{A}\left(\psi_{1}\left(\lambda+p r \alpha_{2}\right), \alpha_{2}\right)$ and $f_{A}\left(\alpha_{1}, \alpha_{2}\right)$.

(i) Determination of $f_{A}\left(\alpha_{1}, 0\right)$ and $f_{A}\left(\psi_{1}(\lambda), 0\right)$.

Taking $\alpha_{2}=0$ in (38), with $\alpha_{1} \neq \psi_{1}\left(\lambda_{1}\right)$, gives

$$
f_{A}\left(\alpha_{1}, 0\right)=\frac{\lambda_{1}}{\lambda}+\frac{\lambda_{2}}{\lambda} \frac{f_{A}\left(\alpha_{1}, 0\right) \psi_{1}(\lambda)-f_{A}\left(\psi_{1}(\lambda), 0\right) \alpha_{1}}{1-\frac{r \alpha_{1}-\eta_{1}\left(\alpha_{1}\right)}{\lambda} \psi_{1}(\lambda)}
$$

which is equivalent to

$$
\left(\lambda_{1}-r \alpha_{1}+\eta_{1}\left(\alpha_{1}\right)\right) f_{A}\left(\alpha_{1}, 0\right)=\lambda_{1}-\lambda f_{A}\left(\psi_{1}(\lambda), 0\right) \frac{\alpha_{1}}{\psi_{1}(\lambda)} .
$$


Setting $\alpha_{1}=\psi_{1}\left(\lambda_{1}\right)$, the left-hand side of (40) becomes zero, and hence, also the right-hand side should be zero, implying $f_{A}\left(\psi_{1}(\lambda), 0\right)=\frac{\lambda_{1}}{\lambda} \frac{\psi_{1}(\lambda)}{\psi_{1}\left(\lambda_{1}\right)}$, and hence,

$$
f_{A}\left(\alpha_{1}, 0\right)=\frac{\lambda_{1}\left(1-\frac{\alpha_{1}}{\psi_{1}\left(\lambda_{1}\right)}\right)}{\lambda_{1}-r \alpha_{1}+\eta_{1}\left(\alpha_{1}\right)}=\frac{1-\frac{\alpha_{1}}{\psi_{1}\left(\lambda_{1}\right)}}{1-\frac{\varphi_{1}\left(\alpha_{1}\right)}{\lambda_{1}}}
$$

Notice that this is also the LST of the workload in $Q_{1}$ just before a gate opening of $Q_{1}$, i.e., after an $\exp \left(\lambda_{1}\right)$ amount of time starting from an empty state, cf. Theorem 4.1 of [7]. This is expected by PASTA.

(ii) Determination of $f_{A}\left(\alpha_{2}, \alpha_{2}\right), f_{A}\left(\psi_{1}\left(\lambda+\operatorname{pr} \alpha_{2}+\eta_{2}\left(\alpha_{2}\right)\right), \alpha_{2}\right)$ and $f_{A}\left(\alpha_{1}, \alpha_{2}\right)$. Introducing the following functions for terms appearing in (38):

$$
\begin{aligned}
A\left(\alpha_{2}\right) & =\frac{\lambda_{1}}{\lambda} \frac{1}{1-\frac{(1-p) r \alpha_{2}-\eta_{1}\left(\alpha_{2}\right)-\eta_{2}\left(\alpha_{2}\right)}{\lambda}}, \\
B\left(\alpha_{2}\right) & =-A\left(\alpha_{2}\right) \frac{\alpha_{2}(1-p)}{\psi_{1}\left(\lambda+p r \alpha_{2}+\eta_{2}\left(\alpha_{2}\right)\right)-p \alpha_{2}}, \\
H(y) & =\frac{\lambda_{2}}{\lambda} \frac{f(y, 0) \psi_{1}(\lambda)-f\left(\psi_{1}(\lambda), 0\right) y}{\left(1-\frac{r y-\eta_{1}(y)}{\lambda}\right) \psi_{1}(\lambda)},
\end{aligned}
$$

one can rewrite (38) as

$$
f_{A}\left(\alpha_{1}, \alpha_{2}\right)=H\left(\alpha_{1}\right)+G\left(\alpha_{2}\right)
$$

where

$$
G\left(\alpha_{2}\right)=A\left(\alpha_{2}\right) f_{A}\left(\alpha_{2}, \alpha_{2}\right)+B\left(\alpha_{2}\right) f_{A}\left(\psi_{1}\left(\lambda+\operatorname{pr} \alpha_{2}+\eta_{2}\left(\alpha_{2}\right)\right), \alpha_{2}\right) .
$$

The decomposition in (45) makes sense as we are observing the system just after gate openings. With probability $\lambda_{i} / \lambda$, the gate opening was at $Q_{i}$, and then $Q_{i}$ has become empty, yielding a term without $\alpha_{i}, i=1,2$. The decomposition form of course implies that $\mathbb{E}\left[Z_{1} Z_{2}\right]=0$, which obviously holds because after each gate opening at least one of the two queues has become empty, and hence, $Z_{1} Z_{2}=0$. This also implies, as is quite intuitive, that $Z_{1}$ and $Z_{2}$ are negatively correlated.

$G\left(\alpha_{2}\right)$ is determined by substituting $\alpha_{1}=\alpha_{2}$, respectively, $\alpha_{1}=\psi_{1}\left(\lambda+\operatorname{pr} \alpha_{2}+\right.$ $\left.\eta_{2}\left(\alpha_{2}\right)\right)$ in (45):

$$
G\left(\alpha_{2}\right)=\frac{A\left(\alpha_{2}\right) H\left(\alpha_{2}\right)+B\left(\alpha_{2}\right) H\left(\psi_{1}\left(\lambda+p r \alpha_{2}+\eta_{2}\left(\alpha_{2}\right)\right)\right)}{1-A\left(\alpha_{2}\right)-B\left(\alpha_{2}\right)},
$$

and hence,

$$
f_{A}\left(\alpha_{1}, \alpha_{2}\right)=H\left(\alpha_{1}\right)+\frac{A\left(\alpha_{2}\right) H\left(\alpha_{2}\right)+B\left(\alpha_{2}\right) H\left(\psi_{1}\left(\lambda+p r \alpha_{2}+\eta_{2}\left(\alpha_{2}\right)\right)\right)}{1-A\left(\alpha_{2}\right)-B\left(\alpha_{2}\right)} .
$$


For completeness we give the LST of the total workload in the two queues, $f_{A}(\alpha, \alpha)$ :

$$
f_{A}(\alpha, \alpha)=\frac{(1-B(\alpha)) H(\alpha)+B(\alpha) H\left(\psi_{1}\left(\lambda+\operatorname{pr} \alpha+\eta_{2}(\alpha)\right)\right)}{1-A(\alpha)-B(\alpha)}
$$

\section{The time-stationary workload LST}

Above we have computed the steady-state joint workload LST of our system just after gate openings. If $f_{B}\left(\alpha_{1}, \alpha_{2}\right)$ is the steady-state joint workload LST just before (any) gate openings, then by PASTA it is also the continuous-time steady-state workload LST. Clearly, one relationship between $f_{A}$ and $f_{B}$ is as follows:

$$
f_{A}\left(\alpha_{1}, \alpha_{2}\right)=\frac{\lambda_{1}}{\lambda} f_{B}\left(\alpha_{2}, \alpha_{2}\right)+\frac{\lambda_{2}}{\lambda} f_{B}\left(\alpha_{1}, 0\right) .
$$

However, this is not enough and in order to compute $f_{B}$ we need to compute the joint LST of the system that starts with distribution having LST $f_{A}$ and ends after an independent exponential time period with parameter $\lambda$. Thus, letting $T \sim \exp (\lambda)$ be independent of everything else, then in an identical manner as for (37) we have (when $r>c_{1}$ ) that

$$
\begin{aligned}
& f_{B}\left(\alpha_{1}, \alpha_{2}\right) \\
& =\frac{f_{A}\left(\alpha_{1}, \alpha_{2}\right)\left(\psi_{1}\left(\lambda+p r \alpha_{2}+\eta_{2}\left(\alpha_{2}\right)\right)-p \alpha_{2}\right)-f_{A}\left(\psi_{1}\left(\lambda+p r \alpha_{2}+\eta_{2}\left(\alpha_{2}\right)\right), \alpha_{2}\right)\left(\alpha_{1}-p \alpha_{2}\right)}{\left(1+\frac{r p \alpha_{2}+\eta_{2}\left(\alpha_{2}\right)-\left(r \alpha_{1}-\eta_{1}\left(\alpha_{1}\right)\right)}{\lambda}\right)\left(\psi_{1}\left(\lambda+r p \alpha_{2}+\eta_{2}\left(\alpha_{2}\right)\right)-p \alpha_{2}\right)} .
\end{aligned}
$$

Therefore, we also have $f_{B}$, the steady-state joint workload LST just before gate openings and at arbitrary epochs.

Determination of moments

It readily follows from (41) that the mean buffer content in $Q_{1}$ right after an arbitrary gate opening is given by

$$
\mathbb{E}\left[Z_{1}\right]=-\left.\frac{d}{d \alpha_{1}} f_{A}\left(\alpha_{1}, 0\right)\right|_{\alpha_{1}=0}=\frac{\eta_{1}^{\prime}(0)-r}{\lambda_{1}}+\frac{1}{\psi_{1}\left(\lambda_{1}\right)} .
$$

$\mathbb{E}\left[Z_{2}\right]$ follows by differentiating the expression in (45) w.r.t. $\alpha_{2}$. Alternatively, we could obtain $\mathbb{E}\left[Z_{1}+Z_{2}\right]$ from (48) and then subtract $\mathbb{E}\left[Z_{1}\right]$. Omitting the messy details, the final result can be written as follows:

$$
\begin{aligned}
\mathbb{E}\left[Z_{2}\right]= & \frac{\eta_{1}^{\prime}(0)-(1-p) r}{\lambda_{2}}+\frac{\lambda_{1}}{\lambda \lambda_{2}} \eta_{2}^{\prime}(0)+\frac{\lambda_{1}^{2}}{\lambda_{2}} \frac{1-p}{\psi_{1}(\lambda)} \\
& +\frac{\lambda_{1}}{\lambda}\left(\mathbb{E}\left[Z_{1}\right]+\frac{\lambda_{1}}{\lambda_{2}}\left(\frac{\psi_{1}(\lambda)}{\psi_{1}\left(\lambda_{1}\right)}-1\right) \frac{1}{\psi_{1}(\lambda)}+\frac{\eta_{1}^{\prime}(0)-r}{\lambda_{1}}\right) \\
& +\frac{\lambda_{1}}{\lambda_{2}} \frac{1-p}{\psi_{1}(\lambda)} H\left(\psi_{1}(\lambda)\right) .
\end{aligned}
$$




\section{Model 3: the shot-noise counterpart of Model 2}

In this section we again consider an ASIP model consisting of two queues $Q_{1}$ and $Q_{2}$ in series, $Q_{i}$ having a gate which opens at independent, $\exp \left(\lambda_{i}\right)$ distributed intervals, for $i=1,2$. If the gate of $Q_{1}$ opens, the buffer content of this queue instantaneously moves to $Q_{2}$; if the gate of $Q_{2}$ opens, the buffer content of this queue instantaneously leaves the system. Again, the two queues receive external input according to two independent Lévy subordinators $J_{i}, i=1,2$. As in Model 2, there is leakage from $Q_{1}$; a fraction $p$ of the leakage from $Q_{1}$ moves to $Q_{2}$ and the rest disappears altogether. So far the model description is the same as for Model 2 in Sect. 3. The special feature of the present model, compared to Model 2, is that in between gate openings, the workload at $Q_{1}$ behaves like a shot-noise process. In a shot-noise process, the workload decreases proportionally to the buffer content, at rate $r x$ when the buffer content equals $x$; this amounts to an exponentially decreasing process, and can be seen as a fluid-type counterpart of an infinite-server queue. It can model situations in which all material that is present in a station is processed simultaneously. In the cell transport model that was described in the previous section, it might be natural to let the processing rate of work (leakage) be proportional to the amount of work (particles present). That is one reason why we wish to investigate this ASIP model with shot-noise-like processing of work.

Material from $Q_{2}$ can leave this queue only when it has a gate opening. For this case, we note that when there is no gate opening before time $t$, then the two buffer contents $Z_{1, x_{1}}(t)$ and $Z_{2, x_{1}, x_{2}}(t)$ evolve as follows:

$$
\begin{aligned}
Z_{1, x_{1}}(t) & =x_{1}+J_{1}(t)-r \int_{0}^{t} Z_{1, x_{1}}(s) d s=x_{1} e^{-r t}+\int_{(0, t]} e^{-r(t-s)} \mathrm{d} J_{1}(s), \\
Z_{2, x_{1}, x_{2}}(t) & =p\left(x_{1}+J_{1}(t)-Z_{1, x_{1}}(t)\right)+x_{2}+J_{2}(t) \\
& =p\left(x_{1}\left(1-e^{-r t}\right)+\int_{(0, t]}\left(1-e^{-r(t-s)}\right) \mathrm{d} J_{1}(s)\right)+x_{2}+J_{2}(t),
\end{aligned}
$$

noting that the fourth equality follows from the second. Indeed, at any time $t$ (before the first gate opening) a fraction $p$ of the difference between $x_{1}+J_{1}(t)$ and the buffer content $Z_{1, x_{1}}(t)$ has moved to $Q_{2}$. Recalling that for nonnegative Borel functions $h$ we have (see, for example, Formula (8) of [5])

$$
\mathbb{E}\left[\mathrm{e}^{-\int_{(0, t]} h(t-s) \mathrm{d} J_{1}(s)}\right]=\mathrm{e}^{-\int_{0}^{t} \eta_{1}(h(s)) \mathrm{d} s},
$$

this implies that

$$
\begin{aligned}
\mathbb{E}\left[\mathrm{e}^{-\alpha_{1} Z_{1, x_{1}}(t)-\alpha_{2} Z_{2, x_{1}, x_{2}}(t)}\right]= & \exp \left(-\alpha_{2} x_{2}-x_{1}\left(\alpha_{1} e^{-r t}+\alpha_{2} p\left(1-e^{-r t}\right)\right)\right. \\
& \left.-\int_{0}^{t} \eta_{1}\left(\alpha_{1} \mathrm{e}^{-r s}+\alpha_{2} p\left(1-e^{-r s}\right)\right) \mathrm{d} s-\eta_{2}\left(\alpha_{2}\right) t\right)
\end{aligned}
$$




$$
\begin{gathered}
=\exp \left(-\alpha_{2} x_{2}-x_{1}\left(\alpha_{1} \mathrm{e}^{-r t}+\alpha_{2} p\left(1-e^{-r t}\right)\right)\right. \\
\left.-\int_{\mathrm{e}^{-r t}}^{1} \eta_{1}\left(\alpha_{1} u+\alpha_{2} p(1-u)\right) \frac{\mathrm{d} u}{r u}-\eta_{2}\left(\alpha_{2}\right) t\right) .
\end{gathered}
$$

Multiplying by $\lambda \mathrm{e}^{-\lambda t}$, where $\lambda=\lambda_{1}+\lambda_{2}$, and integrating give, after the obvious change of variables $v=\mathrm{e}^{-r t}$,

$$
\begin{aligned}
\mathbb{E}\left[\mathrm{e}^{-\alpha_{1} Z_{1, x_{1}}(T)-\alpha_{2} Z_{2, x_{1}, x_{2}}(T)}\right]= & \frac{\lambda}{r} \int_{0}^{1} v^{\frac{\lambda+\eta_{2}\left(\alpha_{2}\right)}{r}-1} \exp \left(-\alpha_{2} x_{2}-x_{1}\left(\alpha_{1} v+\alpha_{2} p(1-v)\right)\right. \\
& \left.-\int_{v}^{1} \eta_{1}\left(\alpha_{1} u+\alpha_{2} p(1-u)\right) \frac{\mathrm{d} u}{r u}\right) \mathrm{d} v
\end{aligned}
$$

where $T \sim \exp (\lambda)$ is independent of everything else.

Remark 2 If, in addition, we assume that $J_{1}$ is a compound Poisson process with arrival rate $\lambda$ and jumps $\sim \exp (\mu)$, then

$$
\eta_{1}(\alpha)=\lambda\left(1-\frac{\mu}{\mu+\alpha}\right)=\frac{\lambda \alpha}{\mu+\alpha}
$$

in which case

$$
\int_{v}^{1} \eta_{1}\left(\alpha_{1} u+\alpha_{2} p(1-u)\right) \frac{\mathrm{d} u}{r u}
$$

can be computed explicitly by observing that

$$
\frac{\eta_{1}\left(\alpha_{1} u+\alpha_{2} p(1-u)\right)}{r u}=\frac{\lambda}{r\left(\mu+p \alpha_{2}\right)}\left(\frac{p \alpha_{2}}{u}+\frac{\mu\left(\alpha_{1}-p \alpha_{2}\right)}{\left(\alpha_{1}-p \alpha_{2}\right) u+\mu+p \alpha_{2}}\right)
$$

and so the integral in (58) becomes

$$
\frac{\lambda}{r\left(\mu+p \alpha_{2}\right)}\left(-p \alpha_{2} \log v+\mu \log \left(\frac{\alpha_{1}+\mu}{\alpha_{1} v+p \alpha_{2}(1-v)+\mu}\right)\right) .
$$

Multiplying by minus one and taking the exponent give

$$
v^{\frac{\lambda p \alpha_{2}}{r\left(\mu+p \alpha_{2}\right)}}\left(\frac{\alpha_{1}+\mu}{\alpha_{1} v+p \alpha_{2}(1-v)+\mu}\right)^{-\frac{\lambda \mu}{r\left(\mu+p \alpha_{2}\right)}} .
$$

We shall now determine the steady-state joint workload LST just before gate openings and just after gate openings. Let $\left(Z_{1, A}, Z_{2, A}\right)$ denote the steady-state workload vector at $Q_{1}$ and $Q_{2}$ just after an arbitrary gate opening, with LST $F_{A}\left(\alpha_{1}, \alpha_{2}\right)$, and let $\left(Z_{1, B}, Z_{2, B}\right)$ denote the steady-state workload vector just before an arbitrary gate opening, with LST $F_{B}\left(\alpha_{1}, \alpha_{2}\right)$. Observe that, if gate 1 just opened, then $Z_{1, A}$ becomes 
zero and $Z_{2, A}$ becomes $Z_{1, B}+Z_{2, B}$, and if gate 2 just opened, then $Z_{1, A}$ becomes $Z_{1, B}$ and $Z_{2, A}$ becomes 0 . Hence, with (again) $\lambda=\lambda_{1}+\lambda_{2}$,

$$
F_{A}\left(\alpha_{1}, \alpha_{2}\right)=\frac{\lambda_{1}}{\lambda} F_{B}\left(\alpha_{2}, \alpha_{2}\right)+\frac{\lambda_{2}}{\lambda} F_{B}\left(\alpha_{1}, 0\right)
$$

From (56), conditioning on $Z_{1, A}=x_{1}, Z_{2, A}=x_{2}$, we have

$$
F_{B}\left(\alpha_{1}, \alpha_{2}\right)=\frac{\lambda}{r} \int_{0}^{1} v^{\frac{\lambda+\eta_{2}\left(\alpha_{2}\right)}{r}-1} \mathrm{e}^{-\frac{1}{r} \int_{v}^{1} \eta_{1}\left(\alpha_{1} u+\alpha_{2} p(1-u)\right) \frac{\mathrm{d} u}{u}} F_{A}\left(\alpha_{1} v+\alpha_{2} p(1-v), \alpha_{2}\right) \mathrm{d} v
$$

We shall first determine $K_{1}\left(\alpha_{1}\right)=F_{B}\left(\alpha_{1}, 0\right)$. Taking $\alpha_{2}=0$ in (63) yields

$$
\begin{aligned}
K_{1}\left(\alpha_{1}\right) & =\frac{\lambda}{r} \int_{0}^{1} v^{\frac{\lambda}{r}-1} \mathrm{e}^{-\frac{1}{r} \int_{v}^{1} \eta_{1}\left(\alpha_{1} u\right) \frac{\mathrm{d} u}{u}} F_{A}\left(\alpha_{1} v, 0\right) \mathrm{d} v \\
& =\frac{\lambda}{r} \int_{0}^{\alpha_{1}} \frac{y^{\frac{\lambda}{r}-1}}{\alpha_{1}^{\frac{\lambda}{r}}} \mathrm{e}^{-\frac{1}{r} \int_{y}^{\alpha_{1}} \eta_{1}(z) \frac{\mathrm{d} z}{z}}\left(\frac{\lambda_{1}}{\lambda}+\frac{\lambda_{2}}{\lambda} K_{1}(y)\right) \mathrm{d} y .
\end{aligned}
$$

Differentiation w.r.t. $\alpha_{1}$ results in a first-order inhomogeneous differential equation:

$$
K_{1}^{\prime}\left(\alpha_{1}\right)=\frac{\lambda_{1}}{r \alpha_{1}}-\left(\frac{\lambda_{1}}{r \alpha_{1}}+\frac{\eta_{1}\left(\alpha_{1}\right)}{r \alpha_{1}}\right) K_{1}\left(\alpha_{1}\right)
$$

whose solution is readily seen to be

$$
K_{1}\left(\alpha_{1}\right)=\alpha_{1}^{-\frac{\lambda_{1}}{r}} \mathrm{e}^{-\frac{1}{r} \int_{0}^{\alpha_{1}} \frac{\eta_{1}(u)}{u} \mathrm{~d} u}\left[C+\frac{\lambda_{1}}{r} \int_{0}^{\alpha_{1}} v^{\frac{\lambda_{1}}{r}-1} \mathrm{e}^{\frac{1}{r} \int_{0}^{v} \frac{\eta_{1}(u)}{u} \mathrm{~d} u} \mathrm{~d} v\right]
$$

The fact that we should have $K_{1}(0)=1$ implies that the term between square brackets should be zero, and hence, $C=0$. One can subsequently quickly verify, by the transformation $w=v / \alpha_{1}$, that

$$
\lim _{\alpha_{1} \downarrow 0} K_{1}\left(\alpha_{1}\right)=\frac{\lambda_{1}}{r} \int_{0}^{1} w^{\frac{\lambda_{1}}{r}-1} \mathrm{~d} w=1 .
$$

We conclude that

$$
K_{1}\left(\alpha_{1}\right)=F_{B}\left(\alpha_{1}, 0\right)=\frac{\lambda_{1}}{r \alpha_{1}} \int_{0}^{\alpha_{1}}\left(\frac{v}{\alpha_{1}}\right)^{\frac{\lambda_{1}}{r}-1} \mathrm{e}^{-\frac{1}{r} \int_{v}^{\alpha_{1}} \frac{\eta_{1}(u)}{u} \mathrm{~d} u} \mathrm{~d} v
$$

By PASTA, this is also the LST of the steady-state workload in $Q_{1}$ at an arbitrary epoch. We next turn to the determination of $K_{2}\left(\alpha_{2}\right)=F_{B}\left(\alpha_{2}, \alpha_{2}\right)$, which, again by PASTA, is the LST of the steady-state total workload in the ASIP system at an arbitrary 
epoch. Taking $\alpha_{1}=\alpha_{2}$ in (63) gives

$$
\begin{aligned}
K_{2}\left(\alpha_{2}\right) & =\frac{\lambda}{r} \int_{0}^{1} v^{\frac{\lambda+\eta_{2}\left(\alpha_{2}\right)}{r}-1} \mathrm{e}^{-\frac{1}{r} \int_{v}^{1} \eta_{1}\left(\alpha_{2} u+\alpha_{2} p(1-u)\right) \frac{\mathrm{d} u}{u}} F_{A}\left(\alpha_{2} v+\alpha_{2} p(1-v), \alpha_{2}\right) \mathrm{d} v \\
& =\frac{\lambda}{r} \int_{0}^{1} v^{\frac{\lambda+\eta_{2}\left(\alpha_{2}\right)}{r}-1} \\
& \times \mathrm{e}^{-\frac{1}{r} \int_{v}^{1} \eta_{1}\left(\alpha_{2} u+\alpha_{2} p(1-u)\right) \frac{\mathrm{d} u}{u}}\left(\frac{\lambda_{1}}{\lambda} K_{2}\left(\alpha_{2}\right)+\frac{\lambda_{2}}{\lambda} K_{1}\left(\alpha_{2} p+\alpha_{2}(1-p) v\right)\right) \mathrm{d} v .
\end{aligned}
$$

Solving for $K_{2}\left(\alpha_{2}\right)$ yields

$$
\begin{aligned}
K_{2}\left(\alpha_{2}\right)= & {\left[1-\frac{\lambda_{1}}{r} \int_{0}^{1} v^{\frac{\lambda+\eta_{2}\left(\alpha_{2}\right)}{r}-1} \mathrm{e}^{-\frac{1}{r} \int_{v}^{1} \eta_{1}\left(\alpha_{2} u+\alpha_{2} p(1-u)\right) \frac{\mathrm{d} u}{u}}\right]^{-1} } \\
& \times \frac{\lambda_{2}}{r} \int_{0}^{1} v^{\frac{\lambda+\eta_{2}\left(\alpha_{2}\right)}{r}-1} \mathrm{e}^{-\frac{1}{r} \int_{v}^{1} \eta_{1}\left(\alpha_{2} u+\alpha_{2} p(1-u)\right) \frac{\mathrm{d} u}{u}} K_{1}\left(\alpha_{2} p+\alpha_{2}(1-p) v\right) \mathrm{d} v .
\end{aligned}
$$

One could subsequently substitute the expression for $K_{1}\left(\alpha_{1}\right)$ as found in (66) in (70). This results in a quite complicated integral, which it seems that one has to evaluate numerically. However, if $p=1$ (so all the leakage goes to $Q_{2}$ ), then (70) simplifies:

$$
\begin{aligned}
K_{2}\left(\alpha_{2}\right) & =\left[1-\frac{\lambda_{1}}{r} \int_{0}^{1} v^{\frac{\lambda+\eta_{2}\left(\alpha_{2}\right)+\eta_{1}\left(\alpha_{2}\right)}{r}-1} \mathrm{~d} v\right]^{-1} \frac{\lambda_{2}}{r} \int_{0}^{1} v^{\frac{\lambda+\eta_{2}\left(\alpha_{2}\right)+\eta_{1}\left(\alpha_{2}\right)}{r}-1} K_{1}\left(\alpha_{2}\right) \mathrm{d} v \\
& =\frac{\frac{\lambda_{2}}{\lambda+\eta_{2}\left(\alpha_{2}\right)+\eta_{1}\left(\alpha_{2}\right)} K_{1}\left(\alpha_{2}\right)}{1-\frac{\lambda_{1}}{\lambda+\eta_{2}\left(\alpha_{2}\right)+\eta_{1}\left(\alpha_{2}\right)}}=\frac{\lambda_{2}}{\lambda_{2}+\eta_{2}\left(\alpha_{2}\right)+\eta_{1}\left(\alpha_{2}\right)} K_{1}\left(\alpha_{2}\right)
\end{aligned}
$$

Remark 3 When the initial workloads at the two stations are $x_{1}, x_{2}$ and when $p=1$, the sum of the workloads at the two stations just before a gate opening is clearly $x_{1}+x_{2}+J_{1}(T)+J_{2}(T)$. Therefore, it is obvious that we necessarily have that

$$
\mathbb{E}\left[\mathrm{e}^{-\alpha Z_{1, x_{1}}(T)-\alpha Z_{2, x_{1}, x_{2}}(T)}\right]=\mathrm{e}^{-\alpha x_{1}-\alpha x_{2}} \frac{\lambda}{\lambda+\eta_{1}(\alpha)+\eta_{2}(\alpha)} .
$$

This agrees with (56) upon setting $\alpha_{1}=\alpha_{2}=\alpha$ (and $p=1$ ) as well as formula (63), which reduces to the following: When we take $p=1$ and $\alpha_{1}=\alpha_{2}=\alpha$,

$$
F_{B}(\alpha, \alpha)=\frac{\lambda}{\lambda+\eta_{1}(\alpha)+\eta_{2}(\alpha)} F_{A}(\alpha, \alpha) .
$$

In combination with (62), this readily agrees with (71).

Acknowledgements The authors are indebted to a referee for some valuable questions and comments. 


\section{References}

1. Asmussen, S.: Applied Probability and Queues, 2nd edn. Springer, New York (2003)

2. Blythe, R.A., Evans, M.R.: Nonequilibrium steady states of matrix product form: a solver's guide. J. Phys. A: Math. Theor. 40, R333-R441 (2007)

3. Bonifacino, J.S., Glick, B.S.: The mechanisms of vesicle budding and fusion. Cell 116, 153-166 (2004)

4. Bonomo, O.L., Reuveni, S.: Occupancy correlations in the asymmetric simple inclusion process. Phys. Rev. E. 100, 042109 (2019)

5. Boxma, O.J., Kella, O., Mandjes, M.R.H.: Infinite-server systems with Coxian arrivals. Queueing Syst. 92, 233-255 (2019)

6. Boxma, O.J., Kella, O., Yechiali, U.: An ASIP model with general gate opening intervals. Queueing Syst. 84, 1-20 (2016)

7. Debicki, K., Mandjes, M.R.H.: Queues and Lévy Fluctuation Theory. Springer, New York (2015)

8. Derrida, B.: An exactly soluble non-equilibrium system: the asymmetric simple exclusion process. Phys. Rep. 301, 65-83 (1998)

9. Golinelli, O., Mallick, K.: The asymmetric simple exclusion process: an integrable model for nonequilibrium statistical mechanics. J. Phys. A: Math. Gen. 39, 12679-12705 (2006)

10. Heckmann, K.: Single file diffusion. In: Kreuzer, F., Slegers, J.F.G. (eds.) Biomembranes 3, pp. 127153. Plenum, New York (1972)

11. Jackson, R.R.P.: Queueing systems with phase-type service. Oper. Res. Q. 5, 109-120 (1954)

12. Jackson, R.R.P.: Random queueing processes with phase-type service. J. R. Stat. Soc. Ser. B (Methodol.) 18, 129-132 (1956)

13. Jenneskens, T.C.W.: Cellular Transport: A Queueing Systems Analysis. Bachelor project thesis, Eindhoven University of Technology (2018)

14. Kella, O., Whitt, W.: Useful martingales for stochastic storage processes with Lévy input. J. Appl. Probab. 29, 396-403 (1992)

15. MacDonald, C.T., Gibbs, J.H., Pipkin, A.C.: Kinetics of biopolymerization on nucleic acid templates. Biopolymers 6, 1-5 (1968)

16. Reuveni, S.: Tandem Stochastic Systems: The Asymmetric Simple Inclusion Process. PhD Thesis, Tel-Aviv University (2014)

17. Reuveni, S., Eliazar, I., Yechiali, U.: Asymmetric inclusion process. Phys. Rev. E 84(041101), 1-16 (2011)

18. Reuveni, S., Eliazar, I., Yechiali, U.: Limit laws for the asymmetric inclusion process. Phys. Rev. E 86(061133), 1-17 (2012)

19. Reuveni, S., Eliazar, I., Yechiali, U.: Asymmetric inclusion process as a showcase of complexity. Phys. Rev. Lett. 109(020603), 1-4 (2012)

20. Reuveni, S., Hirschberg, O., Eliazar, I., Yechiali, U.: Occupation probabilities and fluctuations in the asymmetric inclusion process. Phys. Rev. E 89(042109), 1-23 (2014)

Publisher's Note Springer Nature remains neutral with regard to jurisdictional claims in published maps and institutional affiliations. 